\title{
Scallop hull and its offset
}

\author{
Shuo-Yan Chou, Tony C Woo*, Lin-Lin Chen ${ }^{\dagger}$, Kai Tang ${ }^{\ddagger}$ and \\ Sung Yong Shin ${ }^{\S}$
}

\begin{abstract}
A linear-time algorithm that computes the envelope of the offset of a monotone chain is presented. The scallop hull, an extended notion of the convex hull, of the monotone chain is first computed by using an approach similar to that of the convexhull construction algorithm. The offset of the scallop hull, which yields the desired envelope, can then be computed in linear time from the scallop hull, giving a tool path.
\end{abstract}

Keywords: offsets, machining, inspection

The problem of finding an offset arises in the generation of tool paths for machining a surface on a numerically controlled (NC) machine. When a hemispherical (ballend) cutter is used to create the surface of a workpiece, the centre of the hemispherical tip of the cutter and the resulting surface maintain a constant distance, which equals the radius of the ball-end cutter. The tool path (taken by the centre of the cutter) can therefore be obtained by computing an offset of the desired locus of contact points on the surface of the workpiece.

There are, in general, two types of NC tool path: 'direction-parallel' ${ }^{1}$ paths, and 'contour-parallel' paths. This paper examines the former by taking the following view. Suppose that the representation is a graph surface $z=f_{1}(x, y)$. (A parametric surface $\mathscr{P}(u, v)$, i.e. $x=F(u, v)$, $y=G(u, v), z=H(u, v)$ can be implicitized as $f_{2}(x, y, z)=0$; hence, $z=f_{1}(x, y)$.) Sectioning the surface with a plane parallel to, say, the $x z$ plane gives a curve of intersection (see Figure 1). The offset of this curve yields one of the zigzag tool paths; the subsequent ones are obtained similarly by taking further parallel sections. Note that the tool paths thus generated do not have to correspond to the constant parametric curves. Also, overcut and undercut may occur, since the surface normals are not necessarily in the section plane. This, when cast in the framework of a coordinate-measuring machine (CMM),

Department of Industrial Management, National Taiwan Institute of Technology, Taiwan

*Department of Industrial and Operations Engineering, University of Michigan, 10E Building, 1205 Beal Avenue, Ann Arbor, MI 48109-2117, USA

${ }^{+}$Graduate School of Engineering Technology, National Taiwan Institute of Technology, Taiwan

${ }^{\ddagger} \mathrm{CAD} / \mathrm{CAM}$ Division, Schlumberger Technologies, Ann Arbor, MI, USA

"Department of Computer Science, Korea Advanced Institute of Science \& Technology, 373-1 Kusong-dong, Yusung-gu, Taejon 305-701, Korea Paper received: 6 December 1993. Revised: 14 February 1994 seems not unreasonable. The probe of a CMM is spherical. The manner in which it acquires data is by contact, via small deviations from the computed tool path.

In the literature on $\mathrm{NC}$ tool-path generation, most researchers ${ }^{2-11}$ deal with sculptured surfaces, and are concerned with tool collision (owing to the possible self intersection of the offset). This paper offers a novel concept, the scallop hull (a generalization of the familiar convex hull ${ }^{12}$ ), and applies it to the computation of a collision-free tool path, all in linear time, i.e. in time that is linearly proportional to the number of segments in the input curve. So that this novel idea is unencumbered by analytic or numerical techniques, the input is assumed to be composed of $n$ line segments, a chain. While there is a selection of 3-, 4- and 5-axis NC machines, the simplest of them, the 3-axis machine, is adopted. Because of the limited number of degrees of freedom, the chain must be monotone, i.e. single-valued in the $z$ direction ${ }^{13}$.

The tool path for a monotone chain can be obtained by first computing an initial offset, and then eliminating the portions that cause gouging. The initial offset is the concatenation of the offsets of every individual line segment and vertex in the chain, as shown in Figure $2 a$. The offset of a line segment is simply a parallel line segment at a given distance. The direction is understood to be away from the material side. A vertex can be thought of as a degenerate line segment, a constant-distance offset from which is a circular arc. The extent of the arc is determined by the perpendiculars of the line segments that meet at the vertex (see Figure 3). To eliminate self intersection in the initial offset, two methods are applicable: one utilizes the closest-points Voronoi diagram ${ }^{1,14,15}$ of the chain to identify self intersections of the initial offset, and the other uses an algorithm developed by Hershberger ${ }^{16}$ for constructing the envelope for a set of line segments. Both methods require $\mathrm{O}(n \log n) \mathrm{time}^{17}$.

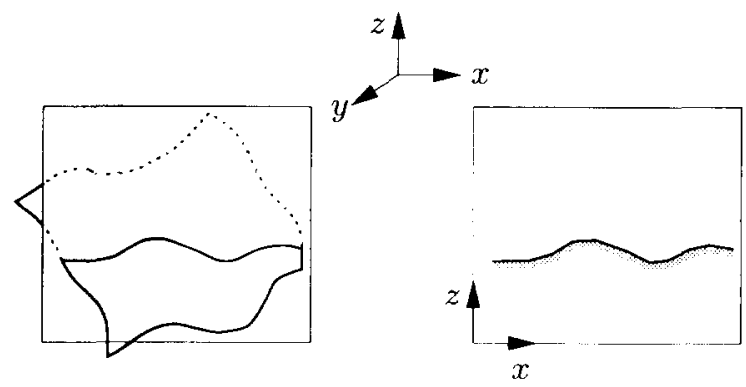

Figure 1 Sectioning a surface and simplifying its geometry 


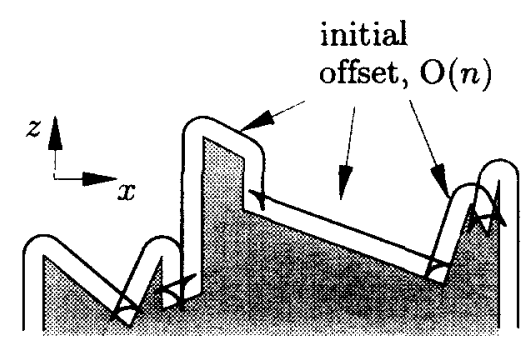

(a)

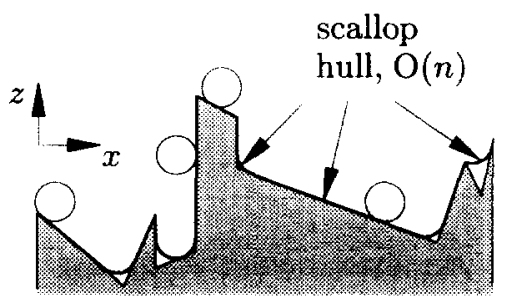

(b) envelop, $\mathrm{O}(n \log n) \quad$ tool

path

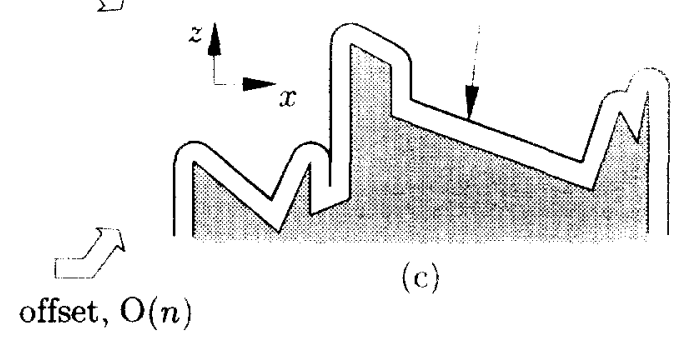

Figure 2 Intersection-free scallop hull

In this paper, rather than dealing with the initial offset, the tool path is computed by offsetting the scallop hull of the monotone chain, which is free of self intersection. The scallop hull is similar to the $\alpha$ hull defined by Edelsbrunner, Kirkpatrick and Seidel ${ }^{18}$ for capturing the 'shape' of a set of points. The parameter $\alpha$ is a real number. When it is positive, the $\alpha$ hull of a set of points is defined as the intersection of all the discs that have radii of $1 / \alpha$, and contain all the points in the set. When $\alpha$ is negative, the $\alpha$ hull is the intersection of all the closed complements of the discs (with radii equal to $-1 / \alpha$ ) that contain all the points in the set. The case of $\alpha<0$ is analogous to a scallop hull for a set of points. The $\alpha$ hull of a set of $n$ points can be computed in $\mathrm{O}(n \log n)$ time $^{18}$.

The scallop hull of a monotone chain is computed by sliding a circle against the boundary of the chain from its nonmaterial side (see Figure $2 b$ ). The monotonicity of the chain facilitates the amortized complexity for backtracking, hence allowing the development of a linear-time algorithm. The scallop hull has three kinds of element: convex vertices, circular arcs substituting for the portions of the chain that cannot be reached by the circle, and the portions of the chain that are in contact with the circle. Notice that there is no concave vertex in a scallop hull, which gives rise to a self-intersecting offset. When the radius of the circle is infinite, the scallop hull of the chain becomes its convex hull.

The tool path is simply the locus of the centre of the circle, which can be stored during the process of constructing the scallop hull. This takes $O(n)$ storage, where $n$ is the number of segments. In this paper, the tool path is computed by offsetting the scallop hull, without a storage cost, as shown in Figure 4. This takes $O(n)$ time, since there is no self intersection in the scallop hull. The total time is $\mathrm{O}(n)+\mathrm{O}(n)=\mathrm{O}(n)$.

\section{PRELIMINARIES}

Let $C=\left\langle v_{1}, v_{2}, \ldots, v_{n}\right\rangle$ be a chain that is monotone with respect to the $x$ axis, and let $C$ be represented by an array of its $n$ vertices. The line segment joining two
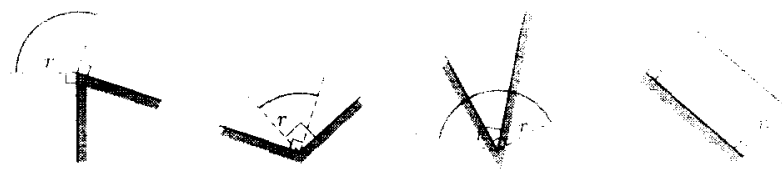

Figure 3 Initial offset of vertex and edge
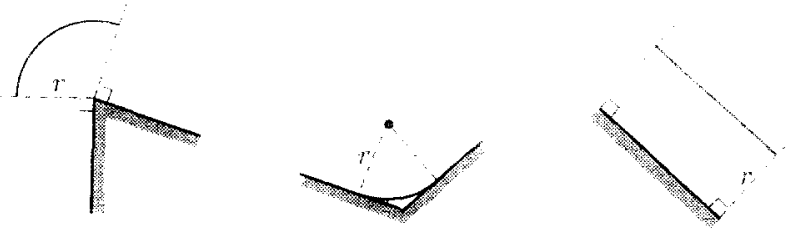

Figure 4 Offset of scallop hull

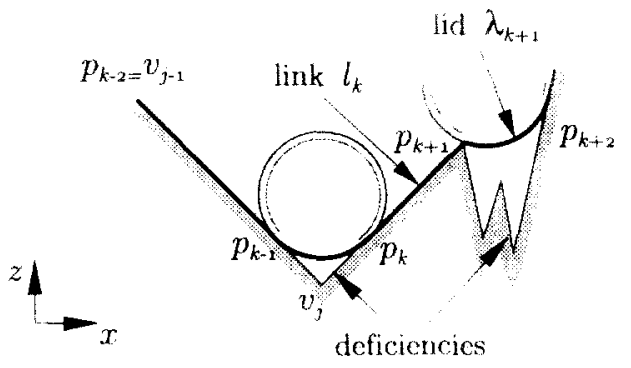

Figure 5 Examples of defined terminologies:

successive vertices $v_{i}$ and $v_{i+1}$ is called an edge of $C$, and it is denoted by $e_{i}$. Assume that $c_{i}$ is directed such that $x_{i} \leqslant x_{i+1}$, for all $1 \leqslant i \leqslant n-1$. Two downward vertical rays with the endpoints at $v_{1}$ and $v_{n}$ are added to the chain $C$ so as to permit the offset of the first and the last vertices.

Sliding a circle of radius $r$ against the left-hand side (the nonmaterial side) of $C$ makes contacts at points $p_{k}$. The portion of $C$ that does not come into contact with the circle is called a deficiency. The two contact points $p_{k-1}$ and $p_{k}$ bounding the deficiency $\left\langle p_{k-1}, v_{j}, p_{k}\right\rangle$, shown in Figure 5 , are the supporting points of the circle. A supporting point can also be at a vertex in the chain. 
such as $p_{k+1}$, shown in Figure 5. The concave upward portion of the circle between two supporting points $p_{k+1}$ and $p_{k+2}$ is called the lid of the deficiency, and it is denoted by $\lambda_{k+1}$. Since $C$ is monotone with respect to the $x$ axis, and since the circle slides against the upper side of $C$, only the lower half of the circle may come into contact with $C$. Let the lower semicircle containing the lid $\lambda_{k+1}$ be denoted as $\pi_{k+1}$. An edge or a partial edge of $C$ which comes into contact with the circle between $p_{k}$ and $p_{k+1}$ (supporting points or vertices of $C$ ) is called a link, and it is denoted by $l_{k}$. The concatenation of lids $\lambda_{i}$ and links $l_{j}$ forms the scallop hull of $C$.

The supporting points of the circle on a pair of edges can be computed analytically. Consider the edges $e_{i}$ and $e_{j}$, as shown in Figure 6. Let the extensions of $e_{i}$ and $e_{j}$ intersect at $q$, and let $\angle v_{i} q v_{j+1}$ denote the angle between the nonmaterial sides of these two lines and let it equal $2 \theta$, where $\theta<90^{\circ}$. Let the distance between $q$ and $v_{i}$ be denoted as $d_{i}$, and likewise for distances to the other vertices. There are three combinations of supporting points, slope-slope, peak-peak and peak-slope, as shown in Figure 6. For a slope-slope pair, as shown in Figure $6 a$, the supporting points $p_{k}$ and $p_{k+1}$ are two interior points of $e_{i}$ and $e_{j}$ such that $d_{k}=d_{k+1}=r / \tan \theta$. The centre and the two endpoints of the lid thus defined can then be computed. For a peak-peak pair, as shown in Figure $6 b$, the centre of the lid lies on the perpendicular bisector of $v_{i}$ and $v_{j+1}$ such that its distances from $v_{i}$ and $v_{j+1}$ equal $r$. For a peak-slope pair, as shown in Figure $6 c$, the centre of the lid lies on the parabolic curve defined with $v_{i}$ as the focus and edge $e_{j}$ as the directrix, such that its distance from $v_{i}$ equals $r$. With the centre of the lid identified, the supporting point $p_{k+1}$ on $e_{j}$ can then be computed.

However, the determination of the combination of supporting points requires the testing of all the possible cases. When $\left(d_{i+1} \cdot \tan \theta\right)<r<\left(d_{i} \cdot \tan \theta\right)$ and $\left(d_{j} \cdot \tan \theta\right)<$ $<r<\left(d_{j+1} \cdot \tan \theta\right)$, only a slope-slope pair of supporting points can be realized. Otherwise, that is, if $r$ and the distances of $q$ to the four vertices of $e_{i}$ and $e_{j}$ do not satisfy the above relationship, whether it is a peak-peak or peak-slope pair can be determined by checking the validity for the rest of the possible cases. Note that vertices $v_{i+1}$ and $v_{j}$ may also be the peak. Additionally, in the proposed algorithm, if $e_{i}$ and $e_{j}$ are not successive edges, supporting points are computed only when the two edges are detected to support the sliding circle. Therefore, by enumeration of all the seven possible pairs, the combination of the supporting points can be verified, and the centre and the endpoints of the lid can then be computed.

\section{CONSTRUCTION OF SCALLOP HULL}

The algorithm for constructing the scallop hull is analogous, in spirit, to the Graham-scan algorithm ${ }^{19}$ for computing the convex hull of a set of points, though it differs in details. The Graham scan starts by sorting the given set of points lexicographically by their polar angles with respect to an arbitrary point in the interior of the convex hull of the set. Interior points are eliminated one by one by comparing three successive points. For the scallop hull, the deficiencies are equivalent to the interior points'. They are eliminated by comparing successive edges of the given monotone chain, and therefore no sorting is required.

Algorithmically, the monotone chain is traversed, and,

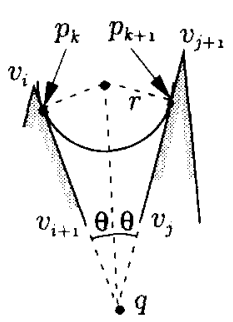

(a)

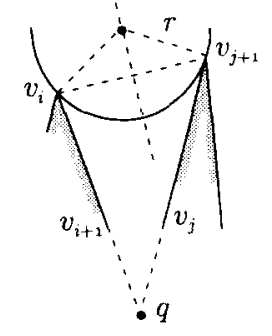

(b)

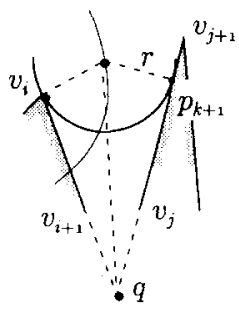

(c)
Figure 6 Pairs of supports; (a) slope-slope, (b) peak-peak, (c) peak-slope

during the process, lids and links of the scallop hull are constructed and/or updated. A current scallop hull is maintained by a stack. Two procedures, forward inclusion and backward exclusion, are executed during the traversal. As suggested by their names, forward inclusion identifies segments (lids and links) that may be pushed into the stack, whereas backward exclusion removes or updates the segments in the stack, whereas backward exclusion removes or updates the segments in the stack. The lids and links remaining in the stack when the algorithm terminates constitute the scallop hull of the chain.

Algorithm (Scallop_Hull)
$\left\langle v_{1}, v_{2}, \ldots, v_{n}\right\rangle:$ a monotone chain
$S$ : the stack maintaining the segments in the current
scallop hull
$k \leftarrow 1, p_{1} \leftarrow v_{1}, \operatorname{TOP}(S) \leftarrow \operatorname{Link}$
for $i=2$ to $n-1$ do
$\quad$ Forward_Inclusion $\left(S, e_{i-1}, e_{i}\right)$
OUTPUT $(S)$

The details in the two procedures are now discussed individually. The Forward_Inclusion procedure first determines whether two successive edges $e_{i-1}$ and $e_{i}$ support a circle. At Step 1 , if the exterior angle of $v_{i}$, formed by $e_{i-1}$ and $e_{i}$, is less than $180^{\circ}$, as shown in Figure $7 a$, the two edges form a support. A lid thus computed is called the active lid. The Backward_Exclusion procedure is then invoked to determine whether the active lid agrees with its preceding lids and links, and to resolve the disagreement if there is any.

If the exterior angle of $v_{i}$, formed by $e_{i-1}$ and $e_{i}$, is greater than or equal to $180^{\circ}$, as shown in Figures $7 b-d$, $e_{i-1}$ and $e_{i}$ do not support the circle. Step 2 begins. If $p_{k} \neq v_{i}$, either the entire edge $e_{i-1}$ (which occurs when the segment obtained in the previous iteration is a link, say $l_{k-1}$, as shown in Figure $7 b$, or the part of $e_{i-1}$ between $v_{i}$ and the supporting point $p_{k}$ (which occurs when the segment obtained in the previous iteration is a lid, say $\hat{\lambda}_{k-1}$, as shown in Figure $7 c$ ), is pushed into the stack as a link $l_{k}$. When $p_{k}=v_{i}$, as shown in Figure $7 d$, no link is generated. This corresponds to Step 2.2.2. However, if $\pi_{k-1}$ intersects $e_{i}$, as shown in Figure $7 e, \lambda_{k-1}$, the lid at the top of the stack, needs to be updated, and the Backward Exclusion procedure is then invoked with the updated $\lambda_{k-1}$ as the active lid. 


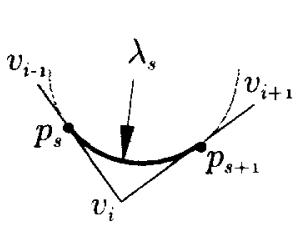

(a)

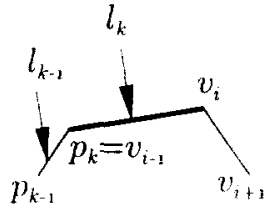

(b)

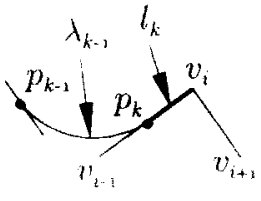

(c)

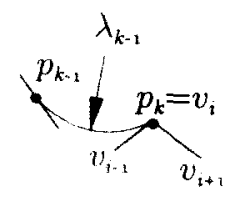

(d)

Figure 7 Cases in Forward Inclusion procedure

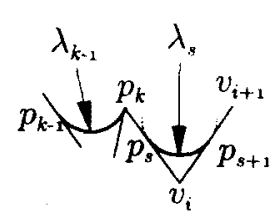

(a)

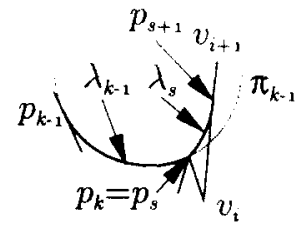

(b)

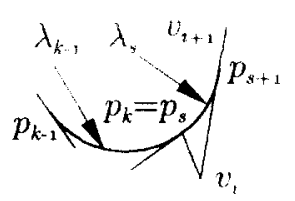

(c)

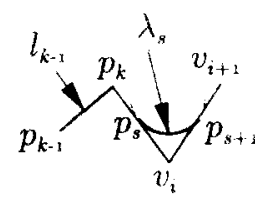

(d)

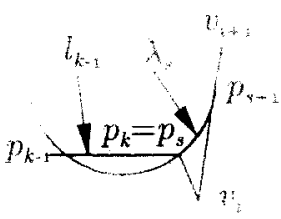

$(e)$

Figure 8 Some cases illustrating Backward Exclusion procedure

\section{Procedure (Forward_Inclusion $\left(S, e_{i-1}, \mathrm{e}_{\mathrm{i}}\right)$ )}

$S$ : the stack maintaining the segments in the current scallop hull

$i_{s}:$ the active lid

(1) if $\angle v_{i-1} v_{i} v_{i+1}<180$ then

Compute $\lambda_{s}$ such that $p_{s} \in e_{i-1}$ and $p_{s+1} \in \ell_{i}$

Backward_Exclusion $\left(S, \lambda_{\mathrm{s}}\right)$

(2) else $\left[\angle v_{i-1} v_{i} v_{i+1} \geqslant 180^{\circ}\right]$

(2.1) if $\operatorname{Top}(S)=$ Link then

$$
\begin{aligned}
& p_{k} \leftarrow l_{i-1}, p_{k+1} \leftarrow l_{i} \\
& \operatorname{PuSH}\left(l_{k}, S\right) \\
& k \leftarrow k+1
\end{aligned}
$$

(2.2) else $\left[\operatorname{TOP}(S)=L i d=\lambda_{k-1}\right]$

$$
\text { (2.2.1) if } \begin{aligned}
p_{k} \neq v_{i} \text { then } \\
\\
p_{k+1} \leftarrow l_{i} \\
\operatorname{pusH}\left(l_{k}, S\right) \\
k \leftarrow k+1
\end{aligned}
$$

(2.2.2) else $\left[p_{k}=v_{i}\right]$

if $\pi_{k-1} \cap e_{i} \neq \varnothing$ then

UPDATE $\left(\lambda_{k-1}\right)$ such that $p_{k} \in e_{i}$

$p_{s} \leftarrow p_{k-1}, p_{s+1} \leftarrow p_{k}$

$k \leftarrow k-1$

Backward_Exclusion $\left(S, \lambda_{s}\right)$

The Backward_Exclusion procedure is responsible for checking whether the active lid $\lambda_{s}$, supported by $e_{i-1}$ and $e_{i}$, agrees with the segment at the top of the stack. TOP $(S)$ can only be a lid or a link. If the segment at the top of the stack is a lid $\lambda_{k-1}$, then the intersection between $\pi_{k-1}$ and $e_{i}$ is checked. If the intersection is empty, the active lid is pushed into the stack; if there exists part of the edge $e_{i-1}$ between $\lambda_{k-1}$ and the active lid, as shown in Figure $8 a$, this partial edge $p_{k} p_{s}$ is pushed into the stack as a link before the active lid is. This corresponds to Step 1.1. If, on the other hand, the intersection between semicircle $\pi_{k-1}$ and the edge $e_{i}$ connecting $v_{i}$ and $v_{i+1}$ is not empty, as shown in Figure $8 b$, a new active lid supported by the edge containing the support $p_{k-1}$ and $\iota_{i}$ is computed. This corresponds to Step 1.2. The Backward Exclusion procedure continues with the new active lid. However, if $\pi_{k-1}$ and the active lid coincide, as shown in Figure $x_{c}$. the lid $\lambda_{k-1}$ is extended to $p_{s+3}$ and the Backward Exclusion procedure terminates.

If the segment at the top of the stack is a link $l_{k-1}$, the side of the active lid on which $l_{k-1}$ lies is determined. AI Step 2.1, if $l_{k-1}$ lies on the concute side of the lower semicircle containing the active lid, the active lid is pushed into the stack. Again, if there exists a part of the edge $i_{i-1}$ between $l_{k-1}$ and the active lid, as shown in Figure $i d$, this partial edge $p_{k} p_{s}$ is pushed into the stack as as link before the active lid is. At Step 2.2. if, on the other hand, a portion of $l_{k-1}$ lies on the convex side of the active lid, as shown in Figure $Y_{e}$, a new active lid supported by the edges containing $p_{k}$; and $c_{i}$ is computed, and the link $l_{k-1}$ is updated accordingly. The Backward_Exclusion procedure then continues with the new active lid.

\section{Procedure (Backward_Exclusion $\left(S, \lambda_{s}\right)$ )}

$S$ : the stack maintaining the segments in the current scallop hull

$\lambda_{s}:$ the active lid

while $\operatorname{rop}(S) \neq$ nil do

(1) if $\operatorname{rOP}(S)=L i d=i_{k \ldots 1}$ then

(1.1) if $\pi_{k-1} \cap e_{i}=\varnothing$ then

if $p_{k} \neq p_{s}$ then

$$
\begin{aligned}
& p_{k+1} \leftarrow p_{s} \\
& \operatorname{PUSH}\left(l_{k}, S\right) \\
& k \leftarrow k+1
\end{aligned}
$$

$p_{k} \leftarrow p_{s}, p_{k+1} \leftarrow p_{s: 1}$

$\operatorname{PUSH}\left(\lambda_{k}, S\right)$

$k \leftarrow k+1$

EXIT

(1.2) else $\left[\pi_{k-1} \cap e_{i} \neq \varnothing\right]$

if $\operatorname{COINCIDE}\left(\pi_{k-1}, \pi_{s}\right)$ then

$i_{k-1} \leftarrow i_{k} \cdots i \lambda_{k}$

$$
\text { EXIT }
$$




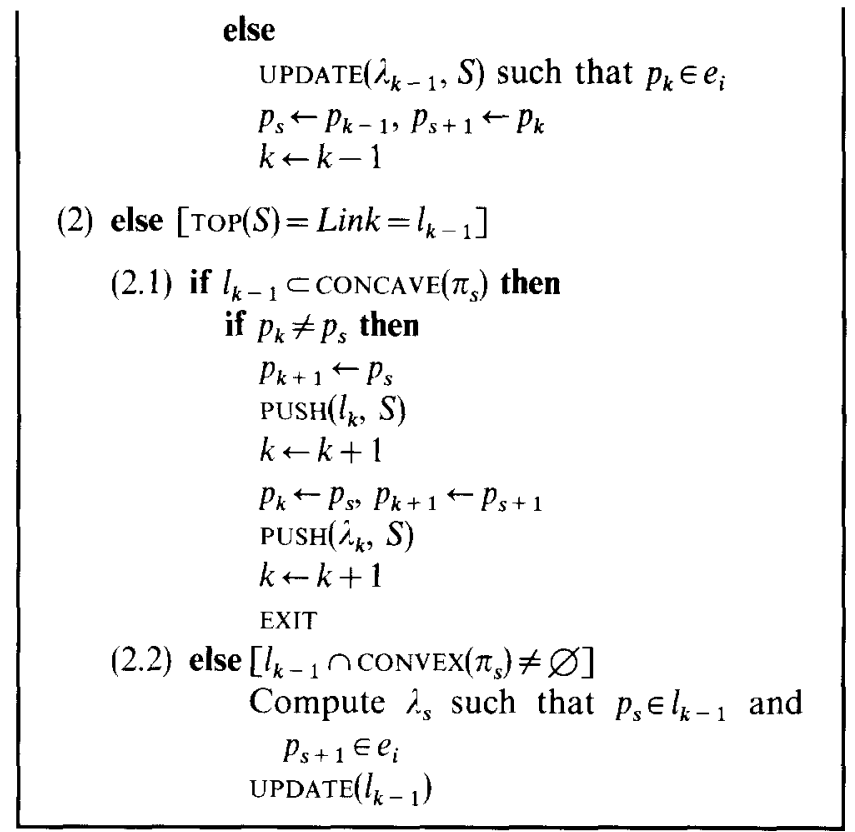

The fact that the algorithm gives a correct scallop hull is now discussed. Forward inclusion computes, for all pairs of successive edges, circular arcs and line segments which may become the lids and links in the scallop hull. When a newly created link conflicts with the lid at the top of the stack (the only possible conflict between a link and the current scallop hull), as shown in Figure $7 e$, a new lid is computed as the active lid, which reduces this case to the case of having a newly created lid. Therefore, backward exclusion only needs to ensure that there is no conflict between an active lid and the current scallop hull. There are two cases of possible conflict. If the top of the stack is a lid $\lambda_{k-1}$, a conflict occurs when $\pi_{k-1}$ intersects $e_{i}$, the second of the pair of successive edges in question, as shown in Figure $8 b$. On the other hand, if the top of the stack is a link $l_{k-1}$, a conflict occurs when a portion of $l_{k-1}$ lies on the convex side of the active lid, as shown in Figure $8 e$. A new active lid is computed in both cases. The following lemma formalizes the fact that, if neither of these two cases occurs, there will be no conflict between the active lid and any lids or links already in the stack.

Lemma: Suppose that an active lid $\hat{\lambda}_{\mathrm{s}}$ is identified in the Forward_Inclusion procedure. If the segment at the top of the stack is (a) a lid $\lambda_{k-1}$ such that $\pi_{k-1}$ does not intersect $e_{i}$, or (b) a link $l_{k-1}$ such that it lies on the concave side of $\dot{\lambda}_{s}$, then $e_{i} \cap \pi_{j-1}=\varnothing$ and $\pi_{k} \cap l_{j-1}=\varnothing$, for all $j<k$.

Proof: (a) Since $C$ is monotonic with respect to the $x$ axis, the $x$ coordinates of the centres of the tentative lids are ordered with respect to the $x$ axis. If $\pi_{k-1}$ does not intersect $e_{i}$, then $\pi_{j-1}$, for $\mathrm{j}<\mathrm{k}$, which is on the concave side of $\pi_{k-1}$, as shown in Figure $9 a$, cannot intersect $e_{i}$ either. On the other hand, by induction, $\pi_{k-1}$ does not intersect $l_{j-1}$, for $j<k-1$, because, otherwise, $\lambda_{k-1}$ would have been updated in an earlier iteration. Moreover, since $x_{j} \leqslant \mathrm{x}_{k-1} \leqslant \mathrm{x}_{s}$ (as shown in Figure $9 b$ ), for $j<k-1$, it is not possible for $\pi_{s}$ to intersect $l_{j-1}$. (b) This part of the lemma can be established using reasoning similar to that for part a.

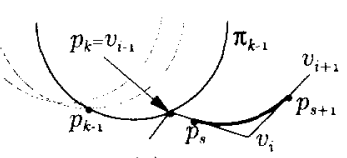

(a)

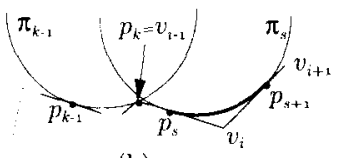

(b)
Figure 9 Termination conditions for Backward_Exclusion procedure

The lemma establishes the stopping condition for the Backward_Exclusion procedure. When the stopping condition is met, the active lid is a lid of the scallop hull for the chain from the beginning of the chain to $e_{i}$. By induction, the Scallop_Hull algorithm described above gives the scallop hull of the monotone chain $C$.

The time complexity of the algorithm for computing the nongouging tool path for a monotone chain is now analysed. There are only $(n+1)$ iterations for forward inclusion, since there are only $(n+1)$ successive pairs of edges (including the two downward vertical rays). Updating the stack, which is the main task in backward exclusion, takes $\mathrm{O}(n)$ time in total, since there are at most $(n-1)$ lids and $(n+2)$ links in the scallop hull, and each segment is stacked or unstacked at most once. Therefore, the time complexity for computing the scallop hull is bounded linearly by the total number of vertices in the chain. The offset of a scallop hull can be computed by concatenating the offset of the links and the vertices in the scallop hull. The offset of a lid is its centre, and it can therefore be ignored. Since the offset of a scallop hull can also be computed easily in $\mathrm{O}(n)$ time, the tool path for a monotone chain can be constructed in linear time. This establishes the following theorem.

Theorem: A non-self-intersecting tool path created by offsetting a monotone chain of $n$ vertices can be computed in $\mathrm{O}(n)$ time.

\section{SUMMARY AND CONCLUSIONS}

The scallop hull of radius $r$ is a generalization of the convex hull (for which $r$ equals infinity). An $\mathrm{O}(n)$ time algorithm for computing the scallop hull of a monotone chain of $n$ edges has been given; the algorithm is therefore optimal.

Applications for the reported work includes $\mathrm{NC}$ machining and CMM inspection. That the mechanisms are 3-axis is underscored by the assumption of the monotonicity of the $2 \mathrm{D}$ data. In $3 \mathrm{D}$, monotonicity is ramified as a terrain surface ${ }^{20}$, for which each point $(x, y, z)$ on the surface is visible from 'above', e.g. from $z=\infty$. Computing the offset for such a terrain surface has obvious use in geographical information systems as well.

\section{REFERENCES}

1 Held, M On the Computational Geometry of Pocket Machining Springer-Verlag (1991)

2 Anderson, $\mathrm{R} \mathrm{O}$ 'Detecting and eliminating collison in $\mathrm{NC}$ machining' Comput.-Aided Des. Vol 10 (1978) pp 231-237

3 Chappel, I T 'The use of vectors to simulate material removed by numerical controlled milling' Comput.-Aided Design Vol 15 (1983) pp 156-158

4 Chen, Y J and Ravani, B 'Offset surface generation and contouring 
in computer-aided design' ASME Trans. J. Mech. Transmissions \& Automat. Des. Vol 109 (1987) pp 133-142

5 Choi, B K, Lee, C S, Hwang, J S and Jun, C S 'Compound surface modelling and machining' Comput.-Aided Design Vol 20 (1988) pp $127-136$

6 Choi, B K and Jun, C S 'Ball-end cutter interference avoidance in NC machining of sculptured surfaces' Comput.-Aided Design Vol 21 (1989) pp 371-378

7 Farouki, R T and Chastung, J A Curves and surfaces in geometrical optics' in Mathematics Methods in CAGD II (1992)

8 Farouki, R T 'Exact offset procedures for simple solids' Comput. Aided Geom. Des. Vol 3 (1985) pp 257-279

9 Hwang, J S 'Interference-free tool-path generation in the NC machining of parametric compound surfaces' Comput.-Aided Des. Vol 24 (1992) pp 667-676

10 Kimmel, $R$ and Bruckstein, A M 'Shape offset via level sets' Comput.-Aided Des. Vol 25 (1993) pp 154-162

11 Kuragaus, $T$ 'Fresdam system for design of aesthetically pleasing free-form objects and generation of collision-free tool paths' Comput.-Aided Des. Vol 24 (1992) pp 573-581

12 Preparata, F P and Shamos, M I Computational Geometry: An Introduction Springer-Verlag (1985)

13 Chen, L L and Woo, T C 'Computational geometry on the sphere for automated machining' ASME J. Mech. Des. Vol 114 No 2 (1992) pp 288-295

14 Lee, D T 'Medial axis transformation of a planar shape' IEEE' Trans. Pattern Anal. \& Mach. Intell. Vol PAMI-4 No 4 (1982) pp 363-369

15 Yang, T C, Shin, S Y and Chwa, K Y 'Rolling dises and their applications' J. Des. \& Manuf. Vol 2 (1992) pp 71-82

16 Hershberger, $\mathrm{J}$ 'Finding the upper envelope of $n$ line segments in $\mathrm{O}(n \log n)$ time' Inf. Proc. Lett. Vol 33 No 4 (1989) pp 169-174

17 Aggarwal, A, Edelsbrunner, H, Raghavan, $\mathrm{P}$ and Tiwari, $\mathrm{P}$ 'Optimal time bounds for some proximity problems in the plane' Inf. Proc. Lett. Vol 42 No 2 (1992) pp 55-60

18 Edelsbrunner, H, Kirkpatrick, D G and Seidel, R 'On the shape of a set of points in the plane' IEEE Trans. Inf. Theor. Vol IT-29 No 4 (1983) pp 551-559

19 Graham, R L 'An efficient algorithm for determining the convex hull of a finite planar set' Inf. Proc. Lett. Vol 1 (1972) pp 132-133

20 Cole, $\mathrm{R}$ and Sharir, $\mathrm{M}$ 'Visibility problems for polyhedral terrains' J. Symbolic Comput. Vol 7 No 1 (1989) pp 11-30
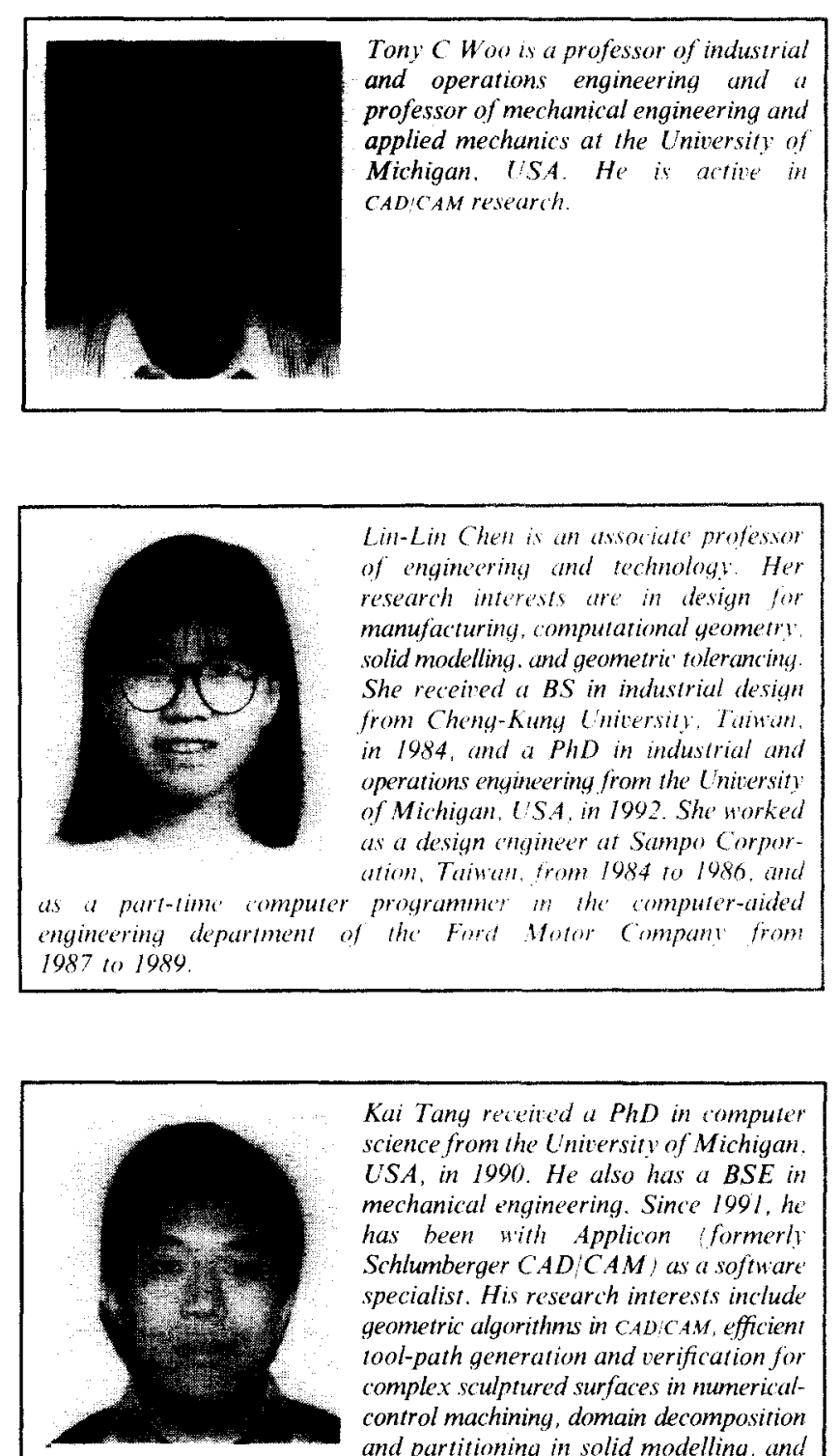

Kai Tany recieived a PhD in computer science from the University of Michigan. USA, in 1990. He also has a BSE in mechanical engineering. Since 1991, he has been with Applicon formerly Schlumberger $C A D(C A M)$ as a software specialist. His research interests include geometric algorithms in CADC CAM, efficient tool-path generation and verification for complex sculptured surfaces in mumericat control machining, domain decomposition and partitioning in solid modelling, and spherical algorithms. His most recent interest is in the efficient computation of swept volumes under various geometric constraints.

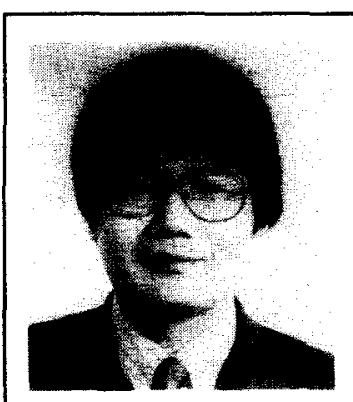

Suny Yond Shin receited a BS in industrial engincering in 1970 from Hanyang Unitersity. Korca, and an MS and a PhD in industrial and operations engineering from the Lniversity of Michigan, LSA, in 1983 and 1986. He is an associate professor in the Department of Computer Science at the Koree Advanced Institute of Science \& Technology. His research interests include computer graphics and compuational geometr. 